\title{
Review: Robot Devices for Gait Rehabilitation
}

\author{
Natasa Koceska \\ Faculty of Computer Science, University "Goce \\ Delcev" - Stip, Macedonia
}

\author{
Saso Koceski \\ Faculty of Computer Science, University "Goce \\ Delcev" - Stip, Macedonia
}

\begin{abstract}
The main motivation of gait rehabilitation is to help a patient recovering from injury, illness or disease, to recover some locomotor abilities in order to promote as much independence as possible in activities of daily living tasks, and to assist the patient in compensating for deficits that cannot be treated medically. However, the amount of hands-on therapy that patients can receive is limited, as economic pressures are inherent in the health care system. Therefore, worldwide efforts are being made to automate locomotor training. Robotic devices has the potential to make therapy more affordable and thus more available for more patients and for more time. This article reviews the most important characteristics and features of the current robot devices for gait rehabilitation, both in clinical use and in the phase of research.
\end{abstract}

\section{General Terms}

Robot rehabilitation.

\section{Keywords}

Robotic systems, exoskeletons, gait rehabilitation, locomotor disabilities.

\section{INTRODUCTION}

Locomotion can be defined as the ability of an organism to independently and safely move both itself and objects from one place to another. It is a complicated repetitious sequence of movement, which requires a neural mechanism, muscles and the skeletal system. Depending on the interaction between the individual and the environment, the neural mechanism directs the musculo-skeletal system to maintain the posture or cause locomotion. In order to ensure static stability during the process of locomotion it is necessary that the centre of mass of the moving organism is vertically projected inside the polygon of support formed by the contact points between its limbs and the ground. Unlike locomotion of the quadrupeds, bipedal human walking is a process of locomotion in which the erect, moving body is supported by first one leg and then the other in order to maintain balance while advancing. During locomotion over a solid substrate humans' limbs are following different patterns of movement. So, gait may be defined as the individual pattern of movement produced as a person walks.

Human gait is measured from heel strike to heel strike, also known as the gait cycle or "one stride." The gait cycle has two phases, the stance (which typically represents $62 \%$ of gait cycle) and the swing phase (which is remaining $38 \%$ of gait cycle). The stance is the period of support, when the foot is in contact with ground, while the swing phase for the same limb, is the non-support phase when the foot is in the air.

Saunders and Inman [1] identified six determinants of gait: pelvic rotation, pelvic tilt, knee and hip flexion, knee and ankle interaction, and lateral pelvic displacement. In normal, level walking, the pelvis rotates about the vertical axis about 4 degrees on the non-weight-bearing side for a total of about 8 degrees over a full cycle, and this rotation increases with gait speed. This rotation reduces the distance the centre of mass must be lifted when the body passes over stance leg and thus reduces the amount of energy required. The second determinant, pelvic tilt, is approximately 5 downward in the coronal plane at normal walking speed and reduces the clearance available for the swing leg. This requires the third determinant, knee flection, in order for the foot to clear during swing phase. The first three determinants work together to reduce the vertical displacement of the centre of mass. Normally, the vertical motion of the centre of mass is about $+/-5 \mathrm{~cm}$.

The irregularities in these determinants determine whether a movement pattern is normal or pathological. Number of factors may contribute to locomotor disabilities and gait dysfunction. It may be due to a medical condition from childhood, such as cerebral palsy, spina bifida, or other developmental disabilities. Or, it may be due to a later injury or illness, such as a traumatic brain injury, incomplete spinal cord injury, stroke and aging.

The number of people with locomotor disabilities is growing permanently as a result of several factors, such as: population growth, ageing and medical advances that preserve and prolong life. Worldwide statistics about locomotor disability show that:

- in Australia: $6.8 \%$ of the Australian population had a disability related to diseases of the musculoskeletal system, which is $34 \%$ of the persons with any kind of disability;

- in USA: there are more than 700.000 Americans who suffer a stroke each year, making it the third most frequent cause of death and the leading cause of permanent disability in the country. 10.000 suffer from traumatic spinal cord injury, and over 250.000 are disabled by multiple sclerosis per year

- in Italy: 1.200.000 people have declared the locomotor disabilities.

\section{Gait rehabilitation}

Gait rehabilitation is very important part of the therapy plan for patients with locomotor dysfunctions in the lower extremities. The goal of gait therapy is to re-train the nervous system, to re-build muscle strength, to improve balance and to re-train kinematics in order to reduce the stresses applied to bones and muscles. On the other hand, it helps the patient to return to the highest level of function and independence possible, while improving the overall quality of life physically, emotionally, and socially.

Gait rehabilitation following neurological injury has been shown to have many therapeutic benefits. Intensive training and exercise may enhance motor recovery or even restore motor function in people suffering from neurological injuries, such as spinal cord injury (SCI) and stroke. It is clinically proved that repetitive practice, strengthens neural connections 
involved in a motor task through reinforcement learning, and therefore enables the patients a faster and better re-learning of the locomotion (walking) [2]. It is also shown that the practice is most effective when it is task-specific [3]. Thus, gait rehabilitation should emphasize repetitive movement patterns in order to maximize motor recovery.

Historically, three different modalities of gait rehabilitation can be individuated:

\section{- conventional manual gait rehabilitation}

partial bodyweight support (PBWS) treadmill gait rehabilitation with manual assistance

\section{- robot-assisted gait rehabilitation}

Conventional manual gait rehabilitation includes specific exercises for strengthening and practicing of one single movement at time. In the clinical setting, a physical therapist will develop exercises that help the patient practice walking. Gait training consists of an individual mixture of methods and exercises from the therapists' toolbox, determined by the possibilities and character of the patient, the preferences and experience of the therapist and his/her background in education. The therapist can use the parallel bars, allowing patients to support themselves using their upper body strength while actively walking. The parallel bars allow patients to gradually put more and more weight on their legs as they recover their ability to walk.

The more sophisticated therapy which over the years has established itself as an effective intervention for improving over-ground walking function, involves practice of stepping on a motorized treadmill with manual assistance and partial bodyweight support (BWS). This kind of therapy makes use of a suspension system to provide proper upright posture as well as balance and safety during treadmill walking. This is accomplished through a harness that removes a controllable portion of the weight from the legs, redistributing it to the trunk and groin, and in the same time allowing free movement of the patients' arms and legs. The movement is provided by a slow moving treadmill. The treadmill constant rate of movement provides rhythmic input which reinforces a coordinated reciprocal pattern of movement. Proper coordination is further assisted by the manual placement of the feet by the therapist. The BWS reduces the demands on muscles, which may enable the patient to work on improving the coordination of the movement while gradually increasing the strength of muscles. The controlled environment may also increase patient confidence by providing a safe way to practice walking. As patients progress, the BWS can be gradually decreased, challenging the patient to assert more postural control and balance [4].

This rehabilitation strategy was derived from research showing the effect of suspending spinalized cats in harnesses over treadmills [5]. From this work with spinalized cats, it was determined that not only a reciprocal locomotor program can be generated at a spinal cord level by central pattern generators, but also, this pattern can be controlled through sensory input. By pulling the stance leg back with the pelvis stabilized in a harness, the treadmill causes extension to the hip of the weight bearing leg, which triggers alternation in the reciprocal pattern controlled by the central pattern generator [6]. Since it was demonstrated by [7] that the quality of locomotion in spinalized cats improved if they were provided a locomotor training program, it seems reasonable to expect that humans with locomotor disabilities might benefit from this type of training.
Clinical studies have confirmed that individuals who receive BWS treadmill training following stroke [8] and spinal cord injury [9] demonstrate improved electromyographic (EMG) activity during locomotion [10], walk more symmetrically [11], are able to bear more weight on their legs.

However, manual assistance, during the BWS treadmill training, relies on physiotherapy procedures which are extremely labour intensive. It is carried out by 2 or 3 physiotherapists, sitting next to the treadmill, and manually guiding patient's legs in coordination with a treadmill. For therapists this training is exhaustive, therefore, training sessions tend to be short and may limit the full potential of the treatment. Manual assistance also lacks repeatability and precision. During the manual therapy it is very difficult for even the most proficient and skilled therapist to provide a proper gait pattern and in that way to maintain high-quality therapy across a full training session of patients, who require this type of attention. Also, manually assisted treadmill training lacks objective measures of patient performance and progress.

A promising solution for assisting patients during rehabilitation process is to design robotic devices. They may enhance traditional treatment techniques by enabling rehabilitation of all the joints together, which is more effective that training only one joint at time; they will provide more precise and repetitive gait trajectory, which was the main problem with the manual therapy; they could accurately measure and track the patient's impairments over the rehabilitation course; they could potentially augment recovery of ambulation in people following neurological injury by increasing the total duration of training and reducing the labour-intensive assistance provided by physical therapists. In the general setting of these robotic systems, a therapist is still responsible for the nonphysical interaction and observation of the patient by maintaining a supervisory role of the training, while the robot carries out the actual physical interaction with the patient.

\section{Design aspects of robot devices for gait rehabilitation}

In the last two decades different types of robot devices have been developed to assist gait training. All these devices must ensure repetitive forward motion while simultaneously helping the patient attain a more precise natural gait. Forward motion can be provided in two ways: by moving the ground or by moving the device.

The latter case implies making a mobile base to follow the patient over ground. The moving-base robot can provide patients to walk in a straight line, to practice starting and stopping, to turn as well as to vary speeds while allowing the patient to experience all the sensory inputs associated with walking. However, these added capabilities of a moving robot base imply that the robot must have large and powerful actuators to drive the robot, large and heavy pelvis actuators, compact base of support to allow indoor manoeuvrability, which will significantly increase the complexity of the design and the cost.

The WalkTrainer (Swortec SA) [12] is an example of an over ground robotic rehabilitation system. It is composed of a walking frame, a pelvic orthosis, a body weight support, two leg orthoses and a real-time controlled electro-stimulation. It is an overground walking reeducation deambulator with the association of pelvic and leg orthoses. 
More sophisticated wearable, exoskeleton suit that can be used for therapeutic activities is the ReWalk (from ARGO Medical Technologies Ltd.) [13]. ReWalk comprises two leg braces with motorized joints and motion sensors, a harness, and a backpack for holding the computer that controls the device and a battery that should last for all day. The system also uses crutches for patient support.

Another exoskeleton device that uses crutches for patient support and safety is Ekso bionics from Berkeley [14]. It is a ready-to-wear, battery-powered, bionic device that is strapped over the user's clothing. Ekso suit is comprised of mechanical braces that wrap around the legs and electric muscles that power walking. A computer on the wearer's back controls the suit, while the patients' arms hold a pair of crutches.

The use of crutches in these cases limits the range of potential users of these devices, because the patients need to have sufficient upper extremity strength to balance with crutches.

The other option (by moving the ground) is more common in practice and is implemented by using a stationary base combined with moving ground platform. This approach has many advantages like attaching multiple structures to the patient simultaneously (for e.g. structure for supporting pelvis actuation mechanism, structure for patient support, etc.), larger footprint support, easy access for the therapist to the patient's body to facilitate the non actuated degrees of freedom, dedicated therapy room space, etc. According the way the moving ground is realized, two major approaches to gait rehabilitation can be individuated: treadmill training and training with a programmable end-effector. In both cases mechanism for patient's body weight suspension is implemented.

The Mechanized Gait Trainer (MGT) group has developed a programmable robot device (Gait Trainer) [15] where the foot is permanently attached to an end-effector that generates a human-like walking pattern. The end-effectors are represented by two foot plates connected to a doubled crank and rocker system. An induction motor drives the cranks via a planetary gear system. The rear ends of the foot plates move forwards and backwards following an ellipsoid-like movement. The backward movement of the footplates simulates the stance phase while the forward movement simulates the swing phase. Different gears can be incorporated to vary stride length and timing. Another foot device developed by MGT group is HapticWalker [16], which is a programmable foot device with permanent foot machine contact, capable of simulating level walking, stairs and stumbling forward motion. The system comprises two 3 DOF robot modules, moving each foot in the sagittal plane. Foot movement along the two base axes in this plane (horizontal, vertical) is performed by linear direct drive motors, which move independently on a common rail, but are connected via a slider-crank system.

Similar foot end-effector mechanism which enables to simulate different terrain types, such as walking on level ground, stairs and slope has been developed by Yoon et al. [17]. The mechanism uses two linear actuators arranged in parallel and connected together by a footplate with revolute joints. The solution is actually a five-bar mechanism with two prismatic actuators which are fixed to the ground, and enables spatial motions of toe and heel parallel to y-axis. Using a slider connected to a timing belt driven by a single servo motor, feet movement in opposite directions at constant speed is enabled.
An important aspect of the robotic stepping devices is that they do not provide active assistance at the ankle joint. They rely on assistance at the hip and knee joints to induce the stepping pattern. This may be a key factor for less impaired patients, because the ankle provides more power than either the hip or knee during normal walking [18].

In treadmill training approach, the patient walks on a treadmill while is attached to or wearing the robotic device. The robot helps the patient to generate the appropriate walking gait trajectory. These systems usually contain system of sensors to evaluate the patient's performance and progress as well as to assure a precise match between the gait velocity and the treadmill speed. Most rehabilitation systems that have been developed (whether commercial or under development) used treadmill training approach (Locomat [19], LocoHelp [20], Reoambulator [21], Walkbot [22], Lopes [23]. Robotic devices used in these rehabilitation systems, typically have exoskeleton structure and according to their assistive capabilities can be classified as active and passive.

Passive exoskeletons assist human users mainly by helping them employ their own power without supplying energy to the user. Several rehabilitation passive devices are reported in the literature. For example the system developed by Walsh et al. [24] uses passive devices like springs to store energy released during negative-work phases of the gait cycle and releases it during the positive-work phases to assist. Another passive system is the leg orthosis designed to assist persons with hemiparesis to walk through elimination of the effects of gravity [25]. It is only composed of links and springs which are completely balancing the effect of gravity over the range of motion. Usually passive devices are perceived by the patients as more comfortable and safer than active ones.

In order to be able to assist lower-limb motions during the gait rehabilitation, the robot devices must be capable of active behaviour. They should assist the elevation of the centre of mass of the body (CoM) during walking in a repetitive way. For active assistance during gait rehabilitation, drive selection is one of the key issues, since human joints require high torques during gait but at the same time, aesthetic issues requires compact and low weight drives. Three different types of actuators can be used: hydraulic cylinders, electrical motors and pneumatic actuators (pneumatic cylinders or artificial muscles). To determine the most suitable actuator we need to determine which degrees of freedom have to be actuated in the exoskeleton, the angle pattern data, forces and power of the lower limb joints.

First hydraulically actuated robot devices for gait assistance presented by Seireg and Grundman [26] and Miyamoto et al . [27] provided an accurate, semi-programmable, repeatable gait system. The device designed by Seireg and Grundman had five degrees of freedom ( 2 at hip, 1 at knee, 2 at ankle), good and simple design, but it was bulky and unusable because of the current state of technology. In general, standard hydraulic cylinders are powerful and capable of being precise, but they are also heavy due to the fluid-filled hoses and actuator cylinders, and the fluid has the potential to leak onto the user.

Using electrical motors would simplify the design and the aesthetic effect should be considerably lower than with the other options. Other important advantages of this solution are the small volume and weight, and the absence of any pressure system and any additional system apart from the batteries. Moreover, including the electrical motors in the exoskeleton wouldn't imply any angle restriction in the joints and they 
could be restricted electrically and mechanically. DC electric motors are used to move patient legs in several gait rehabilitation systems like Lokomat, HapticWalker, GaitTrainer and ReWalk.

The use of the pneumatic actuators is reasonable due to their large power output at a relatively low cost. They are also clean, easy to work with, and lightweight. Moreover, the choice of adopting the pneumatic actuators to actuate the prototype joints is biologically inspired. Indeed, the pneumatic pistons and especially artificial muscles are more similar to the biological muscles with respect to the electric motors. They provide linear movements, and are actuated in both directions, so the articulation structures do not require the typical antagonistic scheme proper of the biological joints. Powered robotic devices for gait rehabilitation do not face an energy density problem. They are not meant to be portable or provide long-term functional replacement. Their purpose is to facilitate motor learning by encouraging proper gait dynamics during locomotor training. As a result, the pneumatic actuators may be considered as the best trade-off between biological inspiration, ease of employment and safe functioning due to the compliance of air, on one hand, and production costs, on the other. Vukobratovic [28] created first and one of the most advanced powered orthosis model. It was using pneumatic actuators at the hip, knee, and ankle to provide assistance in the frontal and sagittal planes. Recently several other solutions were presented: POGO-the pneumatically operated gait orthosis [29], and pneumatically driven exoskeleton devices developed by the research group at the University of L'Aquila [30].

\subsection{Body-weight support system}

One of the key components of robot-assisted gait rehabilitation systems is its body-weight support (BWS) mechanism which is used to off-load a part of the weight of the patient during stance phase, reducing the load that needs to be overcome by the patient and ensuring safety and stability during walking. Research has shown that BWS assisted treadmill-based neural training can significantly improve the rehabilitation outcome [31].

Week muscles of the patient at the beginning of the rehabilitation prevents patients from supporting their own body weight and therefore the amount of body weight to be offloaded is very large (usually $60 \%-80 \%$ of the body weight)[32] at this stage. During the time, as the mobility and strength of the patient improves, the amount of body weight to be offloaded is gradually reduced according to the rehabilitation protocol.

According to the counterpoise systems used, two types of BWS systems can be classified: one is the passive BWS and the other one is the active dynamic BWS.

The passive BWS approach can only statically reduce the weight of the patient. It does not reduce the dynamic load (i.e., the inertial force) on the patient's body while he/she is walking on the treadmill. It may also lead to poor offloading accuracy and dynamic performance due to the added counterweights or springs.

The active BWS approach can help the patient to adapt not only to the static gravitational load but also to the dynamic load. These systems can make the patient feel like having a dynamically reduced mass, regardless the way he moves on the treadmill. This can be achieved by force control capability with acceleration feedback. Dedicated actuator is used to control the position and the tension of the cable (or rope) which suspends the patient's torso. The actuator is usually controlled by a closed-loop approach. Several groups have developed robot devices with BWS systems based on different types of actuators: pneumatic, hydraulic and electromagnetic actuators.

\subsection{Pelvis actuation mechanism}

The fact that three of the six gait determinants are related to the pelvic motion highlights its importance during the gait rehabilitation process. Although initially gait rehabilitation robots were focused on controlling leg movement, in recent studies and devices the importance of the pelvic motion in normal locomotion has also been considered. During unconstrained locomotion, the pelvis performs three translational and three rotational movements, which are tightly coupled to step length and stride parameters. To enable back-drivability of the robot, all un-actuated pelvic degree of freedom must be free to move through their natural range during walking. In addition, the mechanism that actuates the pelvis must also transmit the vertical BWS forces to the patient.

Different concepts for pelvis mechanism are possible. Most of them actuate two degrees of freedom: the vertical and the lateral. They can be realized in three different setups: a) parallelogram that translates in the vertical direction and rotates to actuate the lateral direction, b) Cartesian (orthogonal) arrangement, c) prismatic vertical and rotary lateral motion.

Most of the gait-training devices have focused primarily on controlling leg movement. However, pelvic motion also plays an important role in normal locomotion.

Lokomat allows unrestricted movement in the vertical direction but restricts pelvic rotation, pelvic obliquity, and horizontial translation of the pelvis. The Mechanized Gait Trainer is using a single DOF mechanism to move the torso along fixed horizontal and vertical trajectories and to approximate those achieved during normal stepping. These fixed trajectories cannot be optimal for every patient regardless his stage of recovery. The rehabilitation system developed at the University of L'Aquila has two DOF Cartesian mechanism. However, all these devices are incapable for direct control or recording of pelvic movements.

Because of this, a pelvic orthoses with 6 DOF was design to be used together with the leg orthosis, for the WalkTrainer robot rehabilitation system.

Another pneumatic robotic device, called PAM (Pelvic Assist Manipulator) [29], was designed to measure and manipulate naturalistic pelvic motion during BWS gait training on a treadmill. PAM uses two robots with three DOF actuated by six pneumatic cylinders. This mechanism actuates three translational and two rotational DOF. The main drawback of this mechanism is that the subjects are not encouraged by the device to actively control pelvis movement.

On the other hand Robotic Gait Rehabilitation (RGR) Trainer [33] is using an impedance control strategy and a linear electromagnetic actuator, to apply a force field to correct secondary gait deviations in pelvic motion. It interacts with the patient in a way that mimic the interaction with a therapist.

\subsection{Biomechanical design and safety issues}

Designing an exoskeleton device for gait rehabilitation is a very challenging task. From an engineering perspective, the 
designs must be flexible to allow both upper and lower body motions, once a subject is in the exoskeleton, since walking involves synergy between upper and lower body motions. It must be also a light weight, easy wearable and must guarantee comfort and safety. From a neuro-motor perspective, an exoskeleton must be adjustable to anatomical parameters of a subject. The exoskeleton should be analogous to the human limbs and trunk in the case of joint positions and distribution of degrees of freedom.

Considering these characteristics, the exoskeleton for gait rehabilitation is expected to be ergonomic, highly maneuverable, and technically robust so the wearer can move, bend, and swing from side to side.

The exoskeleton-type systems for gait rehabilitation always work around humans or even touch with them and the conventional safety strategies for industrial robots are not well fit to exoskeleton-type systems. Considering the impact force in a sudden collision, which is the possibility of injury to humans, Ikuta et al. [34] proposed a general safety evaluation method by employing the critical impact force $F_{c}$ as a minimal impact force that causes injury to humans and giving the definition of danger index as the producible impact force $\mathrm{F}$ against $\mathrm{F}_{\mathrm{c}}$ :

$$
\alpha=\frac{F}{F_{c}}, \alpha \geq 0
$$

By using this method, not only the impact force $\mathrm{F}$ but also many types of safety or dangerousness can be evaluated quantitatively.

For ensuring safety, the stop block is important to the system. The joint motion spaces on exoskeletons should be limited in those of human operator. Especially under the abnormal state, the exoskeleton cannot hurt the operator due to its over-scaled joint motion spaces. Generally the stop blocks on intelligent machines can be cataloged into soft and hard stop blocks, which are implemented in software and hardware, respectively.

Many of the developed gait rehabilitation robot systems are putting attention on the safety issues and mechanisms. Lokomat is equipped with an optical light sensor system that monitors the patient's feet; if the patient trips, the Lokomat and treadmill are automatically shut off. The Reoambulator is equipped with safety interlocks, redundant travel limits, variable torque limits, and a safety light array to detect improper foot drop. The Haptic Walker implements foot safety release bindings and adjustable ankle range-of-motion limit switches.

\section{Gait-rehabilitation systems}

Over the last decade, a number of robotic devices for gait rehabilitation have been developed, some of them are commercialized, and some still in the research phase.

The Lokomat (Hocoma AG) is the fist motor driven exoskeleton device that is commercially available. It employs a body weight support suspension system and treadmill. Locomat has four rotary joints that drive hip and knee flexion/extension for each leg. The joints are driven in a gaitlike pattern by precision ball screws connected to DC motors. The patient's legs, strapped into an adjustable aluminum frame, are moved with repeatable predefined hip- and kneejoint trajectories on the basis of a position-control strategy. The computer controlled guidance allows individual adjustments of different gait parameters. Lokomat system enable longer and individually adapted training sessions, offering better chances for rehabilitation, in less time and at lower cost compared to existing manual methods.

Another commercially available gait training device is Gait Trainer. It is a single degree-of-freedom powered machine that drives the feet trough a gait-driven trajectory. Gait Trainer applies the principle of movable footplates, where each of the patients' feet is positioned on a separate footplate whose movements are controlled by a planetary gear system, simulating foot motion walking. Gait Trainer use a servocontrolled motor that sense the patients' effort, and keeps the rotation speed constant. A potential limitation with the Gait Trainer is that the system does not directly control the knee or hip joints, so a manual assistance of one physiotherapist is needed to assist their proper movements. Gait Trainer might not be suitable for non-ambulatory people with weak muscles but only for those that have some degree of control of the knee/hip joints.

Reoambulator (marketed in the US as the 'AutoAmbulator') is a robotic gait training device that integrates Body WeightSupport Treadmill Training (BWSTT) with advanced robotics to help rehabilitate patients who experience problems with ambulation, balance, coordination, posture or stamina. Reoambulator was developed in cooperation with the HealthSouth network of rehabilitation hospitals. The device's synchronized robotic legs contribute to the movement, thereby allowing the patient to walk safely and create normal gait patterns. At the same time, multiple sensors perform continuous monitoring and adjustment of power and speed according to the patient's physical requirements. An interactive display with multiple scene modes works to increase patient motivation and add variation to the repetitive training exercises.

In order to release physiotherapists from their physical strain and to offer a more efficient locomotor training on the treadmill the Lokohelp Group has introduced the LokoHelp gait trainer in the world market. LokoHelp enables the treadmill therapy without manual support by a physiotherapist even at seriously disabled patients. It is an electromechanical gait training device that uses a treadmill and a body weight support system for the patient. The LokoHelp is placed in the middle of the treadmill surface parallel to the walking direction and fixed to the front of the treadmill with a simple clamp. It allows an active walking of the patient, active kneeextension, active impact on the foot sole and active hipextension to improve the treatment results. Inclining the treadmill additionally facilitates the hip-extension, which is necessary to activate the locomotor generators.

The ReWalk is commercially available motorized exoskeleton suit from ARGO Medical Technologies Ltd., that can be used for therapeutic activities. ReWalk comprises light wearable brace support suit, which integrates DC motors at the joints, rechargeable batteries, an array of sensors, and a computer-based control system. ReWalk controls movement using subtle changes in center of gravity, mimics natural gait and provides functional walking speed. A forward tilt of the upper body is sensed by the system, which triggers the first step. Repeated body shifting generates a sequence of steps which allows natural and efficient walking. The ReWalk also sits, stands, allows turning and has the ability to climb and descend stairs. Because it mimics a natural walking motion and requires users to control their body movements, the ReWalk system provides a form of exercise that may be particularly beneficial for wheelchairs users with limited exercise options. 
At the end of 2011, Swortec successfully launched the new high-end device WalkTrainer. It is a unique mobile robotic device with electro-stimulation, allowing upright posture and training with true locomotion for paralysed patients, by closely mimicking natural, voluntary, over-ground walking. This will not only stimulate the re-learning of the functional motor pattern of over-ground walking in an optimal manner, but will also increase patient's motivation, which constitutes a crucial element in the subject's re-education. The WalkTrainer device is composed of a walking frame, pelvic orthosis, a body weight support, two leg orthoses and a real-time controlled electro-stimulation. The device actively assists patients in their movements by correcting their manner of moving and by controlling their equilibrium.

Ekso Bionics has developed and manufactured 'Ekso', the ready-to-wear, battery-powered exoskeleton that helps people with lower-extremity paralysis or weakness to get them standing up and walking. The combination of motors and sensors, along with patient assist with balance and body positioning, allow the user to walk over ground with an efficient reciprocal gait pattern. The torso and leg straps are designed for the user/patient to easily get in and out of the device either on their own or with minimal assistance. A physical therapist actuates steps with a button push. The user progresses from sit to stand and using a walker to walking with crutches. User takes control of actuating his/her steps via buttons on the crutches or walker. The user achieves the next step by moving his/her hips forward and shifting them laterally. The Ekso device recognizes that the user is in the correct position and steps. The Ekso exoskeleton can be set to provide audio feedback when the user achieves the ideal lateral and forward shift required for a step.

Walkbot from P\&S Mechanics is a rehabilitation solution for paraplegic patients that enable effective and systematic gait training. It uses a BWS system and a treadmill. It takes into account the gait patterns of the patient and allows easy walking exercises suitable to the patient's joint function and muscle force. Walkbot has automatically adjustable leg length.

HapticWalker is a gait training device which is still in the research phase. It is programmable footplate machine, with permanent foot machine contact. The system comprises two 3 DOF robot modules, moving each foot in the sagittal plane. Foot movement along the two base axes in this plane (horizontal, vertical) is performed by linear direct drive motors, which move independently on a common rail, but are connected via a slider-crank system. A limitation of the HapticWalker is that the interaction only takes place at the foot sole so that typical poor joint stability of stroke patients cannot be controlled, for example to prevent hyperextension of the knee (similar to the GaitTrainer). Furthermore the cutaneous input at the foot sole with such a system is unnatural, which might disturb training effectivity.

The Biomechatronics Lab at the University of California has developed several robotic devices for locomotor training: a device designed to measure and manipulates human stepping on a treadmill (ARTHuR); the Pneumatically Operated Gait Orthosis (POGO) and the Pelvic Assist Manipulator (PAM). These robotic devices are developed with the aim of providing natural gait movements, using backdrivability and compliance as the starting points for the robot design.

The first gait-related robot prototype was ARTHuR [35], a 2dof robot that makes use of linear electric direct drive (nongeared) motors to precisely apply forces to the leg during stepping. It was designed primarily to conduct research on human motor control of gait and has been successfully used with unimpaired participants to create novel dynamic environments that can enhance motor adaptation.

PAM is a device that can assist the pelvic motion during stepping using BWST, and it's used in combination with POGO- the pneumatically operated gait orthosis [29]. PAM is a 5-dof compliant robot that accommodates natural pelvic movement during walking, and POGO is a leg robot aimed at the goal of being used in the clinic for daily locomotor training rather than just for research, but which also preserves good force control. PAM and POGO's actuators are pneumatic. Recent developments in pneumatic actuators and valves allow them to be considered for applications for which previously only electric motors were suitable. Pneumatic systems are inherently compliant, which enables a softer and more natural interaction with the patient. Moreover, pneumatic actuators offer a higher power-to-weight ratio than electric motors. Their low price could potentially facilitate clinical dissemination.

ALEX (Active Leg Exoskeleton) [36] is a motorized exoskeleton for gait rehabilitation of patients with walking disabilities. It is equipped with linear actuators at hip and knee joints, and force-torque sensors and encoders. While the patient walks on a treadmill a computer display in front of the subject provides visual feedback of the executed gait trajectory during training. A force-field controller is used to apply desired force fields on the moving leg. It provides close to zero impedance when the subject moves on the desired gait and offers higher impedance with deviation.

LOPES (Lower Extremity Powered Exoskeleton) robot is a combination of an exoskeleton robot for the legs and an externally supporting end-effector robot for the pelvis. The joints of the robot (hip, knee) are actuated with Bowden-cable driven series elastic actuators. The forwards and backwards motion is driven by an open-loop force-controllable linear actuator. Impedance control is used as a basic interaction control outline for the exoskeleton.

Pneumatically Driven Exoskeleton at University of L'Aquila is a robotic system for gait training of persons with motor impairment, which is still in the research phase. The system has 10-dof: two on the pelvis level, two for the hips, two for the knee and four for the ankles. It uses 4 double-acting rod pneumatic actuators (two for each leg), that controls the hip and knee joints. Motion of each cylinder's piston is controlled by two pressure proportional valves, connected to both cylinder chambers. Hip and knee angles, are acquired with the rotational potentiometers. The mechanical structure of the shapes and the dimensions of the parts composing the exoskeleton are human inspired and have an ergonomic design. The exoskeleton is positioned on a treadmill, and is physically connected with a space guide mechanism located at pelvis height. Space guide mechanism is connected with the chassis which is equipped with a weight balance system, that provide partial bodyweight unloading while the patient practice stepping on a treadmill. The control strategy has been specifically designed in order to ensure a proper position control guiding patient's legs along a fixed reference gait pattern. 
Table 1. Classification of a current gait rehabilitation systems

\begin{tabular}{|l|l|l|l|l|l|l|}
\hline & \multicolumn{2}{|l|}{$\begin{array}{l}\text { Classification according design aspects } \\
\text { and rehabilitation principles }\end{array}$} & \multicolumn{2}{l|}{$\begin{array}{l}\text { Classification according } \\
\text { actuators type }\end{array}$} & $\begin{array}{l}\text { Use of } \\
\text { BWS } \\
\text { system }\end{array}$ \\
\hline & $\begin{array}{l}\text { Overground/ } \\
\text { Wearable } \\
\text { devices }\end{array}$ & $\begin{array}{l}\text { Treadmill } \\
\text { gait } \\
\text { trainer }\end{array}$ & $\begin{array}{l}\text { Foot-plate } \\
\text { based gait } \\
\text { trainers }\end{array}$ & $\begin{array}{l}\text { Electrical } \\
\text { motors }\end{array}$ & $\begin{array}{l}\text { Pneumatic } \\
\text { cylinders }\end{array}$ & \\
\hline WalkTrainer & $\mathrm{X}$ & & & $\mathrm{X}$ & & \\
\hline ReWalk & $\mathrm{X}$ & & & $\mathrm{X}$ & & \\
\hline Ekso bionics & $\mathrm{X}$ & & & $\mathrm{X}$ & & \\
\hline Gait Trainer & & & $\mathrm{X}$ & $\mathrm{X}$ & & $\mathrm{X}$ \\
\hline HapticWalker & & $\mathrm{X}$ & $\mathrm{X}$ & $\mathrm{X}$ & & $\mathrm{X}$ \\
\hline Locomat & & $\mathrm{X}$ & & $\mathrm{X}$ & & $\mathrm{X}$ \\
\hline LocoHelp & & $\mathrm{X}$ & & $\mathrm{X}$ & & $\mathrm{X}$ \\
\hline Reoambulator & & $\mathrm{X}$ & & $\mathrm{X}$ & & $\mathrm{X}$ \\
\hline Walkbot & & $\mathrm{X}$ & & $\mathrm{X}$ & & $\mathrm{X}$ \\
\hline Arthur & & $\mathrm{X}$ & & $\mathrm{X}$ & & $\mathrm{X}$ \\
\hline POGO, PAM & & $\mathrm{X}$ & & & $\mathrm{X}$ & $\mathrm{X}$ \\
\hline ALEX & & $\mathrm{X}$ & & $\mathrm{X}$ & & \\
\hline Lopes & & $\mathrm{X}$ & & $\mathrm{X}$ & & $\mathrm{X}$ \\
\hline $\begin{array}{l}\text { Exoskeleton } \\
\text { developed at } \\
\text { the University } \\
\text { of L'Aquila }\end{array}$ & & $\mathrm{X}$ & & & $\mathrm{X}$ & $\mathrm{X}$ \\
\hline & & & & & & \\
\hline
\end{tabular}

\section{Conclusion}

Robotic rehabilitation devices have the potential to enhance the impact of physical rehabilitation due to their potential greater availability to patients. It has been shown that robot gait-rehabilitation using treadmill training with partial bodyweight support could restore and maintain some degree of ambulatory capacity in incomplete SCI individuals. Robotic gait training may help to make locomotor training more affordable and thus more available for a longer time and also available in the chronic stages of SCI. Recent studies show that gait training may be beneficial at any time after SCI, even years after the injury.

A number of gait-training robotic systems are already in use in clinics worldwide. Clinical trials have shown that robotic gait training can be as effective as manually assisted training for recovery of locomotor capacity.

In parallel, several groups are working toward improving gait-training robotic technologies. These improvements include enhancing the facilitation of normative, locomotorlike sensory feedback that gait training robots provide. They also include the incorporation of adaptation and the principle of assistance-as-needed that is, the ability of the robots to grade the forces that they apply to the patient. This encourages the patient to contribute to the stepping motion as much as possible, which is likely essential for maximizing locomotor plasticity. Another important contribution of robotic gait training systems will be to provide a controlled and quantified test bed for understanding the physiological principles of locomotor recovery.

\section{References}

[1] Saunders, J.B., Inman, V.T., \& Eberhart, H.D. 1953. The major determinants in normal and pathological gait. Journal of Bone and Joint Surgery, 35A, 543-558.

[2] Henry FM: Specificity vs. generality in learning motor skill. In Classical Studies on Physical Activity. Edited by Brown RC and Kenyon GS. Englewood Cliffs, N.J., Prentice-Hall; 1968:331-340.

[3] Edgerton VR, de Leon RD, Tillakaratne N, Recktenwald MR, Hodgson JA, Roy RR: Use-dependent plasticity in spinal stepping and standing. Advances in Neurology 1997, 72:233-247.

[4] Miller EW, Quinn ME, Seddon PG. Body weight support treadmill and overground ambulation training for two patients with chronic disability secondary to stroke. Phys Ther. 2002;82:53-61.

[5] Visintin M, Barbeau H. The effects of body weight support on the locomotor pattern of spastic paretic patients. Can J Neurol Sci. 1989;16:315-325.

[6] Grillner S. Interaction between central and peripheral mechanisms in the control of locomotion. Prog Brain Res. 1979;50:227-235.

[7] Barbeau H, Rossignol S. Recovery of locomotion after chronic spinalization in the adult cat. Brain Res. 1987;412(1):84-95.

[8] Hesse, S.H., Bertelt, C.B., Schaffrin, A.S., Malezic, M. M., \& Mauritz, K.M. (October 1994), Restoration of gait in non-ambulatory hemiparetic patients by treadmill training with partial body weight support, Arch. Phys. Med. Rehabil. 75, 1087-1093.

[9] Wernig, A.W., Nanassy, A.N. \& Muller, A.M. (1999), Laufband (treadmill) therapy in incomplete paraplegia and tetraplegia, J. Neurotrauma 16, 719-726.

[10] Visintin, M.V., Barbeau, H.B, Bitensky, N.B, \& Mayo, N.M. (1998), Using a new approach to retrain gait in stroke patients through body weight support and treadmill training, Stroke 29,1122-1128.

[11] Hassid, E.H., Rose, D.R., Commisarow, J.C., Guttry, M.G. \& Dobkin, B.D. (1997), Improved gait symmetry in hemiparetic stroke patients induced during body weight supported treadmill stepping, J. Neurol. Rehabil. $11,21-26$.

[12] Stauffer Yves, Mohamed Bouri, Reymond Clavel, Yves Allemand and Roland Brodard2 (2010). A Novel Verticalized Reeducation Device for Spinal Cord Injuries: the WalkTrainer, from Design to Clinical Trials, Robotics 2010 Current and Future Challenges, Houssem Abdellatif (Ed.), ISBN: 978-953-7619-78-7, InTech, Available http://www.intechopen.com/books/robotics-2010current-and-future-challenges/a-novel-verticalizedreeducation-device-for-spinal-cord-injuries.

[13] A.Goffer, "Gait-locomotor apparatus," US patent number 7153242,2006

[14] Ekso [online]. Available: www.eksobionics.com

[15] S. Hesse and D. Uhlenbrock, "A mechanized gait trainer for restoration of gait," Journal of rehabilitation research and development, vol. 37, no. 6,pp. 701-708, 2000.

[16] H. Schmidt, S. Hesse, R. Bernhardt and J. Krüger, .HapticWalker.A Novel Haptic Foot Device., ACM Transactions on Applied Perception, Vol. 2, No. 2, April 2005, Pages 166.180

[17] Jungwon Yoon, Bondhan Novandy, Chul-Ho Yoon, and Ki-Jong Park, "A 6-DOF Gait Rehabilitation Robot with Upper- and Lower-Limb Connections that Allows Walking Velocity Updates on Various Terrains", IEEE Transactions on Mechatronics. 2010

[18] Meinders M, Gitter A, Czerniecki JM. The role of ankle plantar flexor muscle work during walking. Scand J Rehabil Med. 1998;30(1):39-46. 
[19] Colombo G., Joerg M., Schreier R., Dietz V., Treadmill training of paraplegic patients using a robotic orthosis, J. Rehabil. Res. Dev. 17 (2000) 35-42.

[20] S. Freivogel, D. Schmalohr, and J. Mehrholz, "Improved walking ability and reduced therapeutic stress with an electromechanical gait device," Journal of Rehabilitation Medicine, vol. 41, no. 9, pp. 734-739, 2009.

[21] G. R. West, "Powered gait orthosis and method of utilizing same," Patent number 6689 075, 2004.

[22] Walkbot [online] Available: http://www.walkbot.co.kr

[23] 31. J. F. Veneman, R. Kruidhof, Edsko E. G. Hekman, R. Ekkelenkamp, Edwin H. F. Van Asseldonk, and Herman van der Kooij, .Design and Evaluation of the LOPES Exoskeleton Robot for Interactive Gait Rehabilitation., IEEE Transactions on Neural systems and rehabilitation engineering, vol. 15, no. 3, september 2007.

[24] C.J. Walsh, D. Paluska, K. Pasch, W. Grand, A. Valiente, and H. Herr. "Development of a lightweight, underactuated exoskeleton for loadcarrying augmentation", Proceedings of the 2006 IEEE International Conference on Robotics and Automation, pages 3485-3491, 2006.

[25] Aguirre-Ollinger, G., Colgate, J.E., Peshkin, M., Goswami, A. 2007, 'Active-Impedance Control of a Lower-Limb Assistive Exoskeleton', 2007 IEEE 10th International Conference on Rehabilitation Robotics, The Netherlands, June 2007 in Proceedings of the 2007 IEEE 10th International Conference on Rehabilitation Robotics, ed Bart Driessen, Just L. Herder, Gert Jan Gelderblom, IEEE, USA, pp. 188-195.

[26] Seireg A, Grundman JG. Design of a multitask exoskeletal walking device for paraplegics. In: Ghista DN, ed. Biomechanics of Medical Devices. New York: Marcel Dekker, Inc.;1981:569-639.

[27] Miyamoto H, Israel I, Miyamoto H, Mori S, Sano A, Sakurai Y. Approach to a powered orthosis for paralyzed lower limbs . In: ICAR $85 ; 1985$. p . 451-8

[28] Vukobratovic M., Hristic D., Stojiljkovic Z. Development of active anthropomorphic exoskeletons. Medical and biological engineering, January 1974, Volume 12, Issue 1, pp 66-80.
[29] Aoyagi D, Ichinose WE, Harkema SJ, Reinkensmeyer DJ, Bobrow JE,"A robot and control algorithm that can synchronously assist in naturalistic motion during body weight supported gait training following neurologic injury," IEEE Transactions on Neural Systems and Rehabilitation Engineering, 15(3):387-400, 2007.

[30] Natasa Koceska, Saso Koceski, Pierluigi Beomonte Zobel and Francesco Durante (2011). Gait Training using Pneumatically Actuated Robot System, Advances in Robot Navigation, Alejandra Barrera (Ed.), ISBN: 978953-307-346-0, InTech, Available from: http://www.intechopen.com/books/advances-in-robotnavigation/gait-training-using-pneumatically-actuatedrobot-system

[31] Werning A, Muller S. Laufband locomotion with body weight support improved walking in persons with severe spinal cord injuries[J]. Paraplegia, 1992, 30(4): 229 -238.

[32] Frey M, Colombo G, Vaglio M, et al. A novel mechatronic body weight support system[J]. IEEE Trans on Neural Syst and Rehab Eng, 2006, 14(3): 311 -321.

[33] Pietrusinski M., Cajigas I., Mizikacioglu Y., Goldsmith M., Bonato P. and Mavroidis C., "Gait Rehabilitation Therapy Using Robot Generated Force Fields Applied at the Pelvis," Proceedings of the 2010 IEEE Haptics Conference, Waltham, MA, March 25-26, 2010, 401407.

[34] Ikuta, K. and Nokata, M. Safety evaluation method of design and control for human-care robots. Int. J. Robot. Res., 2003, 22, 281-297.

[35] Reinkensmeyer D, Wynne J, Harkema S. "A robotic tool for studying locomotor adaptation and rehabilitation", 2002; Second Joint Meeting of the IEEE EMBS and BMES.

[36] Sai K. Banala, Suni K. Agrawal and John P. Scholz, "Active Leg Exoskeleton (ALEX) for Gait Rehabilitation of Motor-Impaired Patients", Proceedings of the 2007 IEEE 10th International Conference on Rehabilitation Robotics, June 12-15, Noordwijk, The Netherlands. 